\title{
Sufficient Condition and Algorithm for Hamiltonian in 3-Connected 3-Regular Planar Bipartite Graph
}

\author{
Md. Khaliluzzaman \\ Department of Computer Science \& Engineering \\ International Islamic University Chittagong (IIUC) \\ Chittagong, Bangladesh.
}

\author{
Md. Monirul Islam \\ Department of Computer Science \& Engineering \\ International Islamic University Chittagong (IIUC) \\ Chittagong, Bangladesh.
}

\author{
Md.Monjur Hasan \\ Department of Computer Science \& Engineering \\ Chittagong University of Engineering \& Technology (CUET) \\ Chittagong, Bangladesh.
}

\begin{abstract}
A graph $\mathrm{G}(\mathrm{V}, \mathrm{E})$ is said to be Hamiltonian if it contains a spanning cycle. The spanning cycle is called a Hamiltonian cycle of $G$ and $G$ is said to be a Hamiltonian graph. A Hamiltonian path is a path that contains all the vertices in $\mathrm{V}$ (G) but does not return to the vertex in which it began. In this paper, we study Hamiltonicity of 3-connected, 3-regular planar bipartite graph $\mathrm{G}$ with partite sets $\mathrm{V}=\mathrm{M} \cup \mathrm{N}$. We shall prove that $\mathrm{G}$ has a Hamiltonian cycle if $\mathrm{G}$ is balanced with $\mathrm{M}$ $=\mathrm{N}$. For that we present an algorithm for a bipartite graph $\mathrm{K}_{\mathrm{M}, \mathrm{N}}$ where $\mathrm{M}>3, \mathrm{~N}>3$ and $\mathrm{M}, \mathrm{N}$ both are even to possess a Hamiltonian cycle. In particular, we also prove a theorem for $\mathrm{S}$ proper subset ( $\mathrm{M}$ or $\mathrm{N}$ ) of $\mathrm{V}$ the number of components $\mathrm{W}$ $(\mathrm{G}-\mathrm{S})=\mathrm{S}$ implies the graph has a Hamiltonian path.
\end{abstract}

\section{Keywoeds}

Hamiltonian Cycle, bipartite, 3-connected, 3-regular, proper subset, Hamiltonian path.

\section{INTRODUCTION}

A path $P$ of a graph $G$ is a Hamiltonian path if $P$ visits every vertex of $\mathrm{G}$ once. Similarly, a cycle $\mathrm{C}$ is a Hamiltonian cycle if it visits each vertex once. A graph is Hamiltonian if it has a Hamiltonian cycle.

Note that if $\mathrm{C}: \mathrm{U} 1 \rightarrow \mathrm{U} 2 \rightarrow \mathrm{U} 3 \rightarrow \ldots \rightarrow \mathrm{Un}$ is a Hamiltonian cycle, and then so is $\mathrm{Ui} \rightarrow \ldots \mathrm{Un} \rightarrow \mathrm{U} 1 \rightarrow \ldots$ Ui-1 for each $\mathrm{i}[1, \mathrm{n}]$ and thus we can choose where to start a cycle.

Hamiltonian paths and cycles are named after William Rowan Hamilton, who devised a puzzle in which a Hamiltonian cycle or path along polyhedron edges of a dodecahedron was sought. Although, Hamilton solved this particular puzzle, evaluation Hamiltonian cycles or paths in arbitrary graphs are proved expected among the hardest problems of information retrieval $[1,2]$. As a result, instead of complete characterization, most researchers aimed to find sufficient conditions for a graph to process a Hamiltonian cycle or path. In this paper, we focus on 3-connected, 3-regular planar bipartite graph to be Hamiltonian.

The study of Hamiltonian cycles and Hamiltonian paths in general and special graphs has been fueled by practical applications and by the issues of complexity. The problem of finding whether a graph $G$ is Hamiltonian is proved to be NP-
Complete for general graphs [3]. The problem remains NPcomplete [3] (1) if $G$ is planar, cubic, 3-connected, and has no face with less than 5 edges, (2) if $G$ is bipartite, (3) if $G$ is the square of a graph, (4) if a Hamiltonian path for $G$ is given as part of the instance. On the other hand the problem of finding whether a graph $G$ contains a Hamiltonian path is also proved to be NP-Complete for general graphs [3]. Again, it remains NP-complete for conditions (1) and (2) mentioned above. Even the variant, in which either the starting point or the end point or both are specified in the input instance, is also NPComplete. No easily testable characterization is known for Hamiltonian graphs. Nor there exist any such condition to test whether a graph contains a Hamiltonian path or not. This is why tremendous amount of research has been done in finding the sufficient conditions for the existence of Hamiltonian cycles or Hamiltonian paths in graphs. The existing conditions in the literature exploits many graph parameters among which degree of vertices, especially pair wise nonadjacent vertices, is worth-noting. We first present some of the famous degree related conditions below.

To the best of our knowledge, the quest for good sufficient degree based conditions for Hamiltonian cycles or paths dates back to 1952 when Dirac presented the following theorem, where $(G)$ denotes the degree of the minimum degree vertex of the graph $G$.

Theorem 1 (see [4]). If $G$ is a simple graph with $n$ vertices, where $n \geq 3$ and $(G) \geq n / 2$, then $G$ contains a Hamiltonian cycle.

A very useful theorem and quite general (the only restriction is that each vertex should have $|\mathrm{E}| / 2$ adjacent or more) was proved by Dirac:

Throrem [Dirac's Theorem]2.If every vertex of a connected graph with 3 or more vertices is adjacent to at least half of the remaining vertices, then the graph has a Hamilton circuit.

The existence of Hamilton cycles in 2-connected, k-regular graphs have been investigated in $[5,6]$ by various authors. The results of these works show that, except for two kinds of graphs of order $3 k+4$ (they are not 3-connected), all 2 connected, k-regular graphs, $k \geq 14$, on at most $3 k+4$ vertices are Hamiltonian. 
In particular, a set of vertices in a graph is independent if no two of the vertices in the set are adjacent. A graph is cubic if every vertex of the graph has degree three.

Dirac [7] showed that if $G$ is a graph with $m \geq 3$ vertices and if every vertex of $G$ has a degree $\frac{m}{2}$ or more, then $G$ is Hamiltonian. Dirac's work has been extended in [7], but these results all require the existence of vertices of degree at least $\frac{m}{2}$. Avoiding this latter requirement, Gordon [8] recently proved the following:

Theorem 3(see [8]). Let $G$ be a finite graph with $2 n$ vertices in which every vertex has degree at least $n-1$. Then either $G$ is Hamiltonian, $G$ has a subgraph isomorphic to $K_{n+l, n-l} G$ has a subgraph isomorphic to $G_{2 n, b}$ for some $b \leq n$, or $G$ has a subgraph isomorphic to $H$, where $G_{2 n, b}$ and $H$ are precisely defined non-Hamiltonian graphs.

As a consequence of Gordon's theorem, if $\mathrm{n} \geq 3$ and if $\mathrm{C}$ is a 2 -connected graph with $2 \mathrm{n}$ vertices which is regular of degree $\mathrm{n}-1$, then $\mathrm{G}$ is Hamiltonian.

A conjecture which has stood the test of time and which appears to be very difficult to prove was proposed by Barnette (problem 5 in [10]). Barnette suggested that every 3connected cubic bipartite planar graph was Hamiltonian. Very little progress has been made on this problem. One of the few results towards the conjecture can be found in Holton, Manvel and McKay [9].

Theorem 4 (see [9]). Let $\mathrm{G}$ be a 3-connected cubic bipartite planar graph. If $\mathrm{IVGI} \leq 64$, then $\mathrm{G}$ is Hamiltonian.

Taking up the theme regarding the number of Hamiltonian cycles in a Hamiltonian graph, Thomassen [11] has provided a condition for a bipartite Hamiltonian graph to have a second Hamiltonian cycle.

Theorem 5(see [11]). Let $\mathrm{G}$ be a Hamiltonian graph with bipartition $A, B$ and let $H$ be a Hamiltonian cycle in $\mathrm{G}$. If every vertex of $B$ has degree at least 3 , then $\mathrm{G}$ has a Hamiltonian cycle other than $\mathrm{H}$.

A Kuratowski subgraph of $G$ is a subgraph of $G$ that is a subdivision of $K_{5}$ or $K_{3,3}$. A minimal non-planar graph is a non-planar graph such that every proper subgraph is planar.

Theorem(Kuratowski, 1930) 6. A graph $G$ is planar iff $G$ does not contain a subdivision of $K_{5}$ or $K_{3,3}$.

In this paper, we show that every 3-connected, 3-regular planar bipartite graph is Hamiltonian. For that, we show a Lemma, that proves $\mathrm{M}=\mathrm{N}$ for a balanced bipartite graph $\mathrm{G}$ where $\mathrm{M}$ and $\mathrm{N}$ are two partite set. After that we proposed an algorithm that possesses a Hamiltonian cycle from a bipartite graph. Then we have proved a theorem if component is equal to proper subset, and then the graph is Hamiltonian. Here $M$ and $\mathrm{N}$ are considered as a proper subset of $\mathrm{V}$ of $\mathrm{G}$ and $\mathrm{M}$ is equal to $\mathrm{N}$. When $\mathrm{M}$ or $\mathrm{N}$ is deleted from $\mathrm{G}$, then the number of components is equal to proper subset that implies the graph has a Hamiltonian path.

The rest of the paper is organized as follows. In Section 2, we present some preliminary notations and results needed throughout the paper. Section 3 presents our main results. Finally, conclude in Section 4.

\section{PRELIMINARIES}

A graph $\mathrm{G}$ can be defined as a pair $(\mathrm{V}, \mathrm{E})$, where $\mathrm{V}$ is a set of vertices, and $\mathrm{E}$ is a set of edges between the vertices $\mathrm{E} \subseteq\{(\mathrm{u}$, v) $\mid \mathrm{u}, \mathrm{v} \subseteq \mathrm{V}\}$. We consider a graph $\mathrm{G}$, which is 3-connected. A graph is 3 -connected if removal of exactly 3 vertices makes the graph disconnected. The graph is also 3-connected, 3regular, because it contains exactly 3 edges for all of its vertices. We considering $G$ that is 3-connected, 3-regular planar bipartite graph, because it contains no edge crossings and there exist two partite vertex sets ( $\mathrm{M}$ and $\mathrm{N})$ which are considered as the nonempty proper subset of V. A Hamiltonian cycle is a spanning cycle in a graph i.e. a cycle through every vertex and a Hamiltonian path is a spanning path. A graph containing a Hamiltonian cycle is said to be Hamiltonian. It is clear that every graph with a Hamiltonian cycle has a Hamiltonian path but the converse is not necessarily true [12].

An independent set of a graph $G=(V, E)$ is a set of vertices $V^{\prime} \subseteq V$ such that all pairs of vertices $u, \mathrm{v} \in V^{\prime}$ are nonadjacent in $G$. A graph can be decomposed into independent sets in the sense that the entire vertex set of the graph can be partitioned into pair wise disjoint independent subsets. Such independent subsets are called partite sets or simply parts. A graph is said to be a $k$-partite graph, if its vertex set can be decomposed into $k$ partite sets but not fewer. So, a bipartite graph is a graph that can be decomposed into two partite sets but not fewer. Similarly, a tripartite graph is a graph that can be decomposed into three partite sets but not fewer. A 1-partite graph is the same as an independent set or an empty graph.

One often writes $G=(\mathrm{M} \cup \mathrm{N}, E)$ to denote a bipartite graph whose partite sets are $\mathrm{M}$ and $\mathrm{N}$. If $|\mathrm{M}|=|\mathrm{N}|$ or $\mathrm{M}=\mathrm{N}$, that is, if the two partite sets have equal cardinality, then $G$ is called a balanced bipartite graph. On the other hand, if || $\mathrm{M}|-| \mathrm{N} \| \leq 1$, then we say that $G$ is a semi-balanced bipartite graph. Note that, by definition, a balanced bipartite graph is also a semibalanced bipartite graph. It is easy to see that, for a bipartite graph $G$ to possess a Hamiltonian path, $G$ must be semibalanced. Similar to the notation used for bipartite graphs, a tripartite graph with partite sets $\mathrm{M}, \mathrm{N}$, and $\mathrm{P}$ may be denoted by $G=(\mathrm{M} \cup \mathrm{N} \cup \mathrm{P}, E)$.

A nonempty set is a set containing one or more elements. Any set other than the empty set $(\phi)$ is therefore a nonempty set. A proper subset $S^{\prime}$ of a set $S$, denoted $S^{\prime} \subset S$, is a subset that is strictly contained in $\mathrm{S}$ and so necessarily excludes at least one member of $S[13,14]$. The empty set is therefore a proper subset of any nonempty set. A component must be defined by a nonempty set. A component is the maximal sub-graph in which a path exists from every node to every other. A connected graph has only one component.

\section{MAIN RESULT}

In this section we present our main results. We first state and prove the following useful Lemma.

Lemma 1. If $G(V, E)$ is a planar bipartite graph of $M$ and $N$ distinct set of vertices $(M>3, N>3$ and $M, N$ both are even), where $G$ is 3-connected 3-regular, then $M=N$ and $G$ is Hamiltonian.

Proof. Suppose two different set of vertices $M=\left\{a_{1}, a_{2}, a_{3}\right.$, $\left.\ldots \ldots, a_{n}\right\}$ and $N=\left\{b_{1}, b_{2}, b_{3}, \ldots . ., b_{n}\right\}$. As it is three regular all vertices in $\mathrm{M}$ and $\mathrm{N}$ sets are of degree three. Thus $\mathrm{a}_{1}$ degree is three, so $\mathrm{a}_{1}$ needs three vertex from $\mathrm{N}$ set's for its connection. Also $b_{1}$ needs three vertices from $M$ sets. For that all vertices 
need three edges from $\mathrm{N}$ and $\mathrm{M}$ sets. But it is not possible to generate a 3-connected 3-regular planar bipartite graph with $\mathrm{M}=3$ and $\mathrm{N}=3$ because $\mathrm{K}_{3,3}$ is not planar (Theorem 6). We need at least 4 vertices in each of set $\mathrm{M}$ and $\mathrm{N}$.

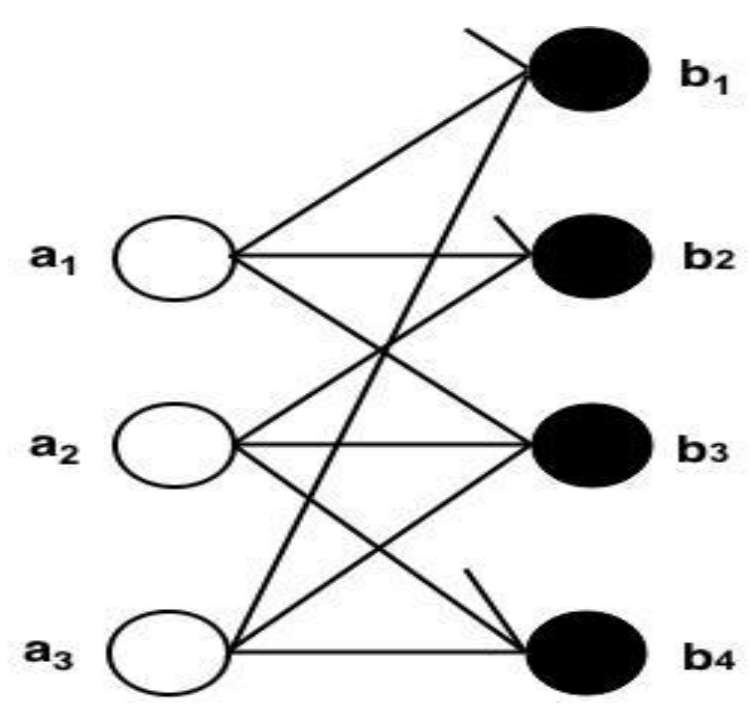

Fig1: A $\mathbf{K}_{3,4}$ bipartite graph.

In undirected graph $\mathrm{u}$ and $\mathrm{v}$ are connected, if it contains a path between $\mathrm{u}$ to $\mathrm{v}$. Suppose, $\mathrm{M}$ has 3 and $\mathrm{N}$ has 4 nodes. As the graph is 3- connected 3-regular, $\mathrm{M}$ contains $3 \times 3=9$ and $\mathrm{N}$ contains $4 \times 3=12$ edges. Here $N$ has extra 3 edges which can't participate in path shown in Figure 1. For path connection N set need extra one vertex for connecting extra 3 edges from $M$ set.

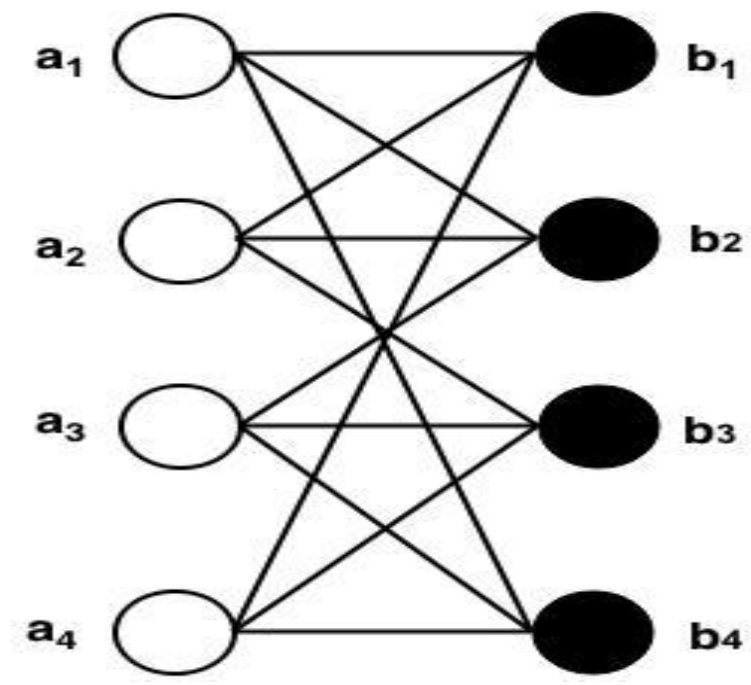

Fig. 2: 3-Connected 3-Regular bipartite Graph.

If $\mathrm{M}$ and $\mathrm{N}$ both have 4 nodes, then $\mathrm{M}$ and $\mathrm{N}$ both contains $4 \times 3=12$ edges shown in Figure 2. To create paths in 3connected 3-regular bipartite graph one set's nodes total degree is same with another set's nodes. When two set's total nodes will be same, then two set's total degree will be same. So there will be $\mathrm{M}=\mathrm{N}$.
Figure 2 is a non-planar bipartite graph. We surely need a formal algorithm to represent non-planar graph as a planar graph with Hamiltonian cycle.

In this algorithm, we consider a bipartite graph $\mathrm{K}_{\mathrm{M}, \mathrm{N}}$ where $\mathrm{M}>3, \mathrm{~N}>3$ and $\mathrm{M}, \mathrm{N}$ both are even.

\section{Algorithm: Hamiltonian_Cycle}

Input: A bipartite graph $\mathrm{K}_{\mathrm{M}, \mathrm{N}}$, Where $\mathrm{M}$ and $\mathrm{N}$ are both even bipartite set and $\mathrm{M}>3, \mathrm{~N}>3$.

Output: A planar Hamiltonian cycle.

Step 1. Partition $M$ into $M_{i}$ and $M_{j}$ where $\mathrm{i}$ is $1<=\mathrm{i}<=\frac{M}{2}$ and

$$
\mathrm{j} \text { is } \frac{M}{2}+1<=\mathrm{j}<=\mathrm{M}
$$

Step 2. Partition $\mathrm{N}$ into $\mathrm{N}_{\mathrm{k}}$ and $\mathrm{N}_{\mathrm{L}}$ where $\mathrm{k}$ is $1<=\mathrm{k}<=\frac{N}{2}$ and

$$
\mathrm{L} \text { is } \frac{N}{2}+1<=\mathrm{L}<=\mathrm{N}
$$

Step 3. For each $\mathrm{i}, 1<=\mathrm{i}<=\frac{M}{2}$ draw all node(s) vertically in odd position.

For each $\mathrm{k}, 1<=\mathrm{k}<=\frac{N}{2}$ draw all node(s) vertically in even position.

Step 4. For each $\mathrm{j}, \frac{M}{2}+1<=\mathrm{j}<=\mathrm{M}$

draw all node(s) vertically in even position parallel to first vertical line drawn in step3.

For each $\mathrm{L}, \frac{N}{2}<=\mathrm{L}<=\mathrm{N}$

draw all node(s) vertically in odd position parallel to first vertical line drawn in step3.

Step 5. For each $\mathrm{i}, 1<=\mathrm{i}<=\mathrm{M}$

draw line $i$ to $j$ both vertically and horizontally where $j$ is neighbor of $i$ and $j € N$.

Step 6. Draw line (i,k) and (j,L) with

$$
\mathrm{i}=1, \mathrm{k}=\frac{N}{2}, \mathrm{j}=\mathrm{M} \text { and } \mathrm{L}=\frac{N}{2}+1 \text {. }
$$

Step 7. Planar Hamiltonian Cycle.

This algorithm represents a non-planar graph to be planar Hamiltonian cycle.

Figure 3 is drawn according to the proposed algorithm from Figure 2. 


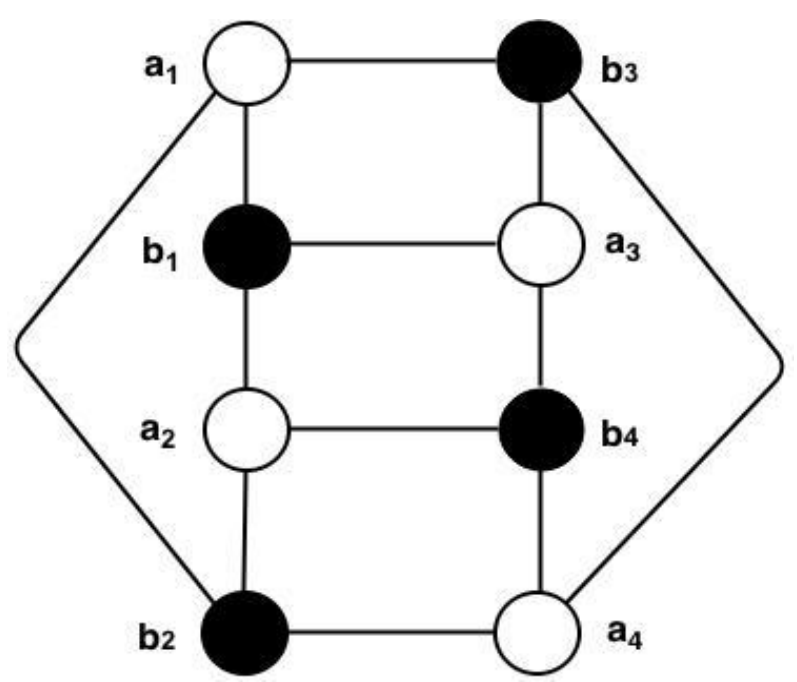

Fig. 3: A 3- connected 3-regular planar bipartite graph.

Now, we present the following sufficient condition.

Theorem 7. If a bipartite graph $G$ is Hamiltonian and $M=N$, where $M$ and $N$ are distinct set of vertices, then for every nonempty proper subset $S$ of $V$ the number of component $W$ $(G-S)=S$.

Proof. Let, G (V, E) be a 3-connected 3-regulat planar bipartite graph, so $\mathrm{M}$ and $\mathrm{N}$ are distinct set of vertices of graph $\mathrm{G}$, then $\mathrm{V}=\mathrm{M}+\mathrm{N}$ and $\mathrm{M}, \mathrm{N}$ are nonempty proper subset of $\mathrm{V}$.

Deleting $\mathrm{S}$ from V of G we found $\mathrm{W}$ (G-S) where $\mathrm{S}$ is the nonempty proper subset of $\mathrm{V}$ and $\mathrm{W}(\mathrm{G}-\mathrm{S})$ are the number of components. For Hamiltonian $\mathrm{S}$ will be distinct set of vertices.

If $\mathrm{M}$ is removed from graph $\mathrm{G}$, then number of components is equal to $\mathrm{N}$ because $\mathrm{G}$ is a bipartite graph of $\mathrm{M}, \mathrm{N}$ nonempty proper distinct subset. Since, in graph $\mathrm{G}, \mathrm{M}=\mathrm{N}$, the number of components is equal to $\mathrm{N}$ or $\mathrm{M}$ which is equal to proper subset $\mathrm{S}$. If $\mathrm{M}$ or $\mathrm{N}$ is deleted from $\mathrm{V}$, then the number of components will be the same with the deleting vertices. So, Component is equal to Proper subset (W $(\mathrm{G}-\mathrm{S})=\mathrm{S})$, hence, the graph $\mathrm{G}$ is Hamiltonian.

In this section, we consider two cases on Theorem 7.

Case 1. In Figure 4 has two different set $M=\{a 1$, a2, a3, a4, $\mathrm{a} 5, \mathrm{a} 6\}$ and $\mathrm{N}=\{\mathrm{b} 1, \mathrm{~b} 2, \mathrm{~b} 3, \mathrm{~b} 4, \mathrm{~b} 5, \mathrm{~b} 6\}$ with every node degree is three, so the graph is three regular and it is three connected bipartite graph. In graph $G$ there is no edge crossing, so, the graph $\mathrm{G}$ is 3-connected 3-reguler planar bipartite graph. Here $\mathrm{M}$ and $\mathrm{N}$ are even and greater than three. Two proper subsets $\mathrm{M}$ and $\mathrm{N}$ (white and black) are exist in this graph. Since, $\mathrm{V}=\mathrm{M}+\mathrm{N}$ and bipartite graph is balanced $(\mathrm{M}=\mathrm{N})$, if we delete one proper subset that is the one partite set $(\mathrm{N}$ or $\mathrm{M})$, then another partite set $(\mathrm{M}$ or $\mathrm{N})$ which is another nonempty proper subset will be remaining in the graph. So, the number of components (W $(\mathrm{G}-\mathrm{S})$ ) is equal to proper subset (S). Thus, graph is Hamiltonian according to proposed theorem.

Case 2. In Figure 5 exist three connected two even bipartite set $\mathrm{M}, \mathrm{N}$ where $\mathrm{M}>3, \mathrm{~N}>3$ and $\mathrm{M}=\mathrm{N}$. Here, every vertices degree is three and there is no edge crossing in graph G, so, the graph $\mathrm{G}$ is 3-connected 3-reguler planar bipartite graph. Two proper subsets $\mathrm{M}$ and $\mathrm{N}$ (white and black) are exist in the graph G. If one partite set (M) is deleted, in graph exist another partite set $(\mathrm{N})$ because of $\mathrm{V}=\mathrm{M}+\mathrm{N}$.

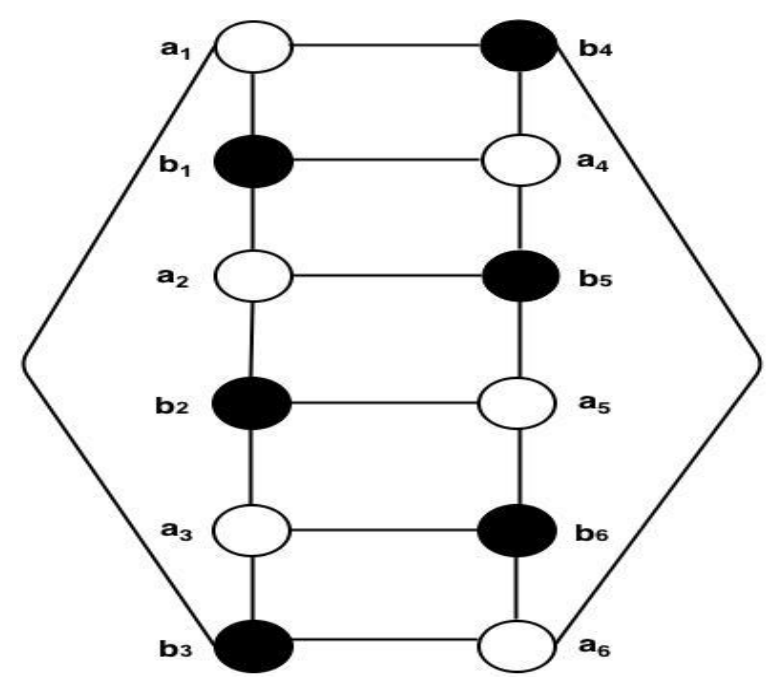

Fig. 4: $K_{6,6}$ 3- connected 3-regular planar bipartite graph.

Since, in graph $\mathrm{G}, \mathrm{M}=\mathrm{N}$, the number of components (W (G$\mathrm{S})$ ) is equal to the proper subset (S) which implies the graph is Hamiltonian.

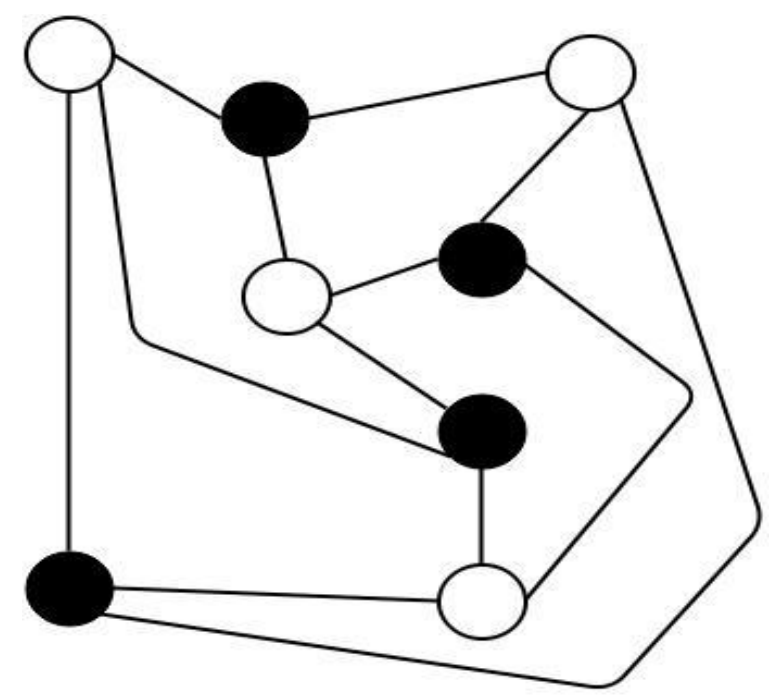

Fig. 5: $\mathrm{K}_{4,4}$ 3- connected 3-regular planar bipartite graph.

\section{CONCLUSION}

In this paper, we present a sufficient condition and algorithm for a 3-connected, 3-regular planar bipartite graph to process a Hamiltonian cycle. For that we proved a Lemma to show partite set of elements are equal hence the graph is balanced and proposed an algorithm that processed a graph to be planar and Hamiltonian where partite set of elements are even and greater than 3 . The algorithm is run in the polynomial time. We also proved a theorem to satisfy that deleting any one partite set of elements which is proper subset of bipartite graph equal to number of components since, partite set of elements are equal. Thus the components are equal to proper subset, and then the graph is Hamiltonian.

Our proposed algorithm is worked for a 3-connected, 3regular planar bipartite graph where partite set of elements are must be even and greater than 3. Anyone can develop an algorithm that works for any number of partite set of elements. 


\section{REFERENCES}

[1] M. R. Garey and D. S. Johnson, Computers and Intractability: A Guide to the Theory of NPCompleteness, W. H. Freeman, New York, NY, USA, 1979.

[2] M. Sohel Rahman, M. Kaykobad, and Jesun Sahariar Firoz, "New Sufficient Conditions for Hamiltonian Paths," The Scientific World Journal, vol. 2014, Article ID 743431, 6 pages, 2014. doi:10.1155/2014/743431.

[3] M.R. Garey and D.S. Jhonson, Computers and Intractability: A Guide to the Theory of NP Completeness, W.H. Freeman and Company, New York.

[4] G.A. Dirac, Some Theorems on Abstract Graphs, Proc. Lond. Math. Soc. 2 (1952), pp 69-81.

[5] P. ErdBs and A.M. Hobbs, Hamilton cycles in regular graphs of moderate degree, J. Combin. Theory Ser. B 23 (1977) 139-142.

[6] Y.-J. Zhu, Z.-H. Liu and Z.-G. Yu, 2-connected kregular graphs on at most $3 \boldsymbol{k}+3$ vertices to be Hamiltonian, J. Systems Sci. Math. Sci. 6 (1) (1986) 36-49 and (2) 1986) 136-145.
[7] G. A. Dirac, Some theorems on abstract graphs. Proc. London Math. Sot., Ser. 3, 2 (1952) 69-81.

[8] L. Gordon, Hamiltonian circuits in graphs with many edges. Unpublished report, Sydney University, Australia.

[9] D.A. Holton, B. Manvel, and B.D. McKay, Hamiltonian cycles in cubic 3- connected bipartite planar graphs, 1. Comb. Th. B, 38 (3) (1985), 279-297.

[10] D. Barnette, Conjecture 5, Recent Progress in Combinatorics, Academic Press, New York, (1969), 343.

[11] C. Thomassen, A Theorem on Paths in Planar Graphs, J. Graph Theory, 7 (1983), 169-176.

[12] Graph Theory with Application in chapter-4. J. A Bondyan.

[13] L. de la Torre, Investigations of Barnette's Graphs, Undergraduate Thesis, Dept. of Math., Univ. of California, Davis, 2005.

[14] G. Chartrand, "Introduction of Graph Theory", New York :Dover, 1985 pp. 29. 